\title{
THE ELM SURVEY. II. TWELVE BINARY WHITE DWARF MERGER SYSTEMS*
}

\author{
Mukremin Kilic ${ }^{1,6}$, Warren R. Brown ${ }^{1}$, Carlos Allende Prieto ${ }^{2,3}$, M. A. Agüeros ${ }^{4}$, Craig Heinke ${ }^{5}$, and S. J. Kenyon ${ }^{1}$ \\ ${ }^{1}$ Smithsonian Astrophysical Observatory, 60 Garden St., Cambridge, MA 02138, USA; mkilic@ cfa.harvard.edu \\ 2 Instituto de Astrofísica de Canarias, E-38205, La Laguna, Tenerife, Spain \\ ${ }^{3}$ Departamento de Astrofísica, Universidad de La Laguna, E-38205, La Laguna, Tenerife, Spain \\ ${ }^{4}$ Department of Astronomy, Columbia University, 550 West 120th Street, New York, NY 10027, USA \\ ${ }^{5}$ Department of Physics, University of Alberta, 11322-89 Avenue, Edmonton, AB, T6G 2G7, Canada \\ Received 2010 August 10; accepted 2010 October 25; published 2010 December 22
}

\begin{abstract}
We describe new radial velocity and X-ray observations of extremely low-mass white dwarfs (ELM WDs, $\sim 0.2 M_{\odot}$ ) in the Sloan Digital Sky Survey Data Release 4 and the MMT Hypervelocity Star survey. We identify four new short period binaries, including two merger systems. These observations bring the total number of short period binary systems identified in our survey to 20 . No main-sequence or neutron star companions are visible in the available optical photometry, radio, and X-ray data. Thus, the companions are most likely WDs. Twelve of these systems will merge within a Hubble time due to gravitational wave radiation. We have now tripled the number of known merging WD systems. We discuss the characteristics of this merger sample and potential links to underluminous supernovae, extreme helium stars, AM CVn systems, and other merger products. We provide new observational tests of the WD mass-period distribution and cooling models for ELM WDs. We also find evidence for a new formation channel for single low-mass WDs through binary mergers of two lower mass objects.
\end{abstract}

Key words: binaries: close - stars: low-mass - white dwarfs

Online-only material: color figures

\section{INTRODUCTION}

Before the recent discovery of the six short period binary WD systems containing $\sim 0.2 M_{\odot}$ extremely low mass white dwarfs (ELM WDs; Kilic et al. 2010b, 2010c; Mullally et al. 2009; Marsh et al. 2010; Kulkarni \& van Kerkwijk 2010; Badenes et al. 2009; Steinfadt et al. 2010; Kawka et al. 2010), there were only six double WD systems known to have short enough orbital periods to merge within a Hubble time (see Nelemans et al. 2005, and references therein). Even though the Supernovae Ia Progenitor Survey (SPY) identified $\approx 100$ new double degenerate systems from a sample of 1000 WDs observed using the Very Large Telescope, Napiwotzki et al. (2004) identify only eight merger candidates (including both WD + WD and sdB + WD systems) from the SPY and the literature. This fraction (8 out of 1000) is relatively small compared with the fraction of merger systems ( 6 out of 8 ) among the ELM WDs in the literature (Kilic et al. 2007b, 2009, 2010b, 2010c).

Low-mass $\left(M<0.45 M_{\odot}\right)$ He-core WDs are formed when a companion strips the outer envelope from a post main-sequence star before the star reaches the tip of the red giant branch and ignites helium. They are usually found in close binaries, mostly double degenerate systems (Marsh 1995). However, about half of the low-mass WDs in the field do not show any radial velocity variations, indicating that they are single (Maxted et al. 2000; Napiwotzki et al. 2007). Kilic et al. (2007c) argue that these single low-mass WDs may come from old metal-rich stars that truncate their evolution prior to the helium flash from severe mass loss. They estimate a binary fraction of $50 \%$ for $\sim 0.4 M_{\odot}$ WDs. However, they predict that the binary fraction rises to $100 \%$ for $\sim 0.2 M_{\odot}$ ELM WDs, since such extreme mass loss

\footnotetext{
* Based on observations obtained at the MMT Observatory, a joint facility of the Smithsonian Institution and the University of Arizona.

6 Spitzer Fellow.
}

rates are not expected even for the most metal-rich stars in the Galaxy.

\subsection{The ELM Survey}

To study the binary fraction of ELM WDs and to find future merger systems, we have undertaken an MMT survey of all previously identified ELM WDs from the SDSS DR4 area (Eisenstein et al. 2006; Liebert et al. 2004) and the Hypervelocity Star survey of Brown et al. (2006). The latter sample is described in a companion paper by Brown et al. (2010a). Part of the SDSS DR4 sample was discussed earlier in Kilic et al. (2010b). Here, we present radial velocity observations of seven more ELM WD candidates from the Sloan Digital Sky Survey (SDSS) sample. The combined sample contains 23 WDs. We also report on Chandra X-ray observations of three ELM WDs to search for X-ray evidence of milli-second pulsar (MSP) companions.

While almost all SDSS ELM WDs show radial velocity variations, none show evidence of main-sequence or neutron star companions in the optical, radio (Agüeros et al. 2009a), or X-ray (this paper and Agüeros et al. 2009b). Hence, they are almost certainly binary WD systems. Out of the 20 short period binary systems in our sample, 12 have merger times shorter than a Hubble time. Our ELM survey has now tripled the number of known merging WD systems. Based on our sample and previously known short period binary WD systems, we study the mass-period distribution and future evolutionary prospects, including the connections to unusual stellar populations and underluminous supernovae ( $\mathrm{SNe}$ ).

Our paper is organized as follows. In Section 2 we discuss our radial velocity and X-ray observations. In Section 3 we present an analysis of the optical spectroscopy and X-ray data and the nature of the companions. In Section 4 we discuss the sample characteristics of ELM WDs, including the binary fraction, mass-period distribution, merging systems, and the 
Table 1

X-ray Observations of ELM WDs

\begin{tabular}{ccccccc}
\hline \hline $\begin{array}{c}\text { Name } \\
\text { (SDSS) }\end{array}$ & ObsID & $\begin{array}{c}\text { Expos. } \\
(\mathrm{ks})\end{array}$ & $\begin{array}{c}\text { Dist. } \\
(\mathrm{kpc})\end{array}$ & $\begin{array}{c}N_{\mathrm{H}} \\
\left(\mathrm{cm}^{-2}\right)\end{array}$ & $\begin{array}{c}\text { Count Rate } \\
\left(\mathrm{counts} \mathrm{s}^{-1}\right)\end{array}$ & $\begin{array}{c}L_{X} \\
\left(\mathrm{erg} \mathrm{s}^{-1}\right)\end{array}$ \\
\hline J105353.89+520031.0 & 9963 & 20.50 & 1.08 & $1.0 \times 10^{20}$ & $<2.2 \times 10^{-4}$ & $<9.5 \times 10^{28}$ \\
J123410.36-022802.8 & 9964 & 5.50 & 0.78 & $2.2 \times 10^{20}$ & $<8.4 \times 10^{-4}$ & $<2.0 \times 10^{29}$ \\
J092345.59+302805.0 & 9965 & 1.03 & 0.27 & $2.0 \times 10^{20}$ & $<4.5 \times 10^{-3}$ & $<1.3 \times 10^{29}$ \\
\hline
\end{tabular}

Notes. $99 \%$ confidence X-ray count rate upper limits for three ELM WDs from Chandra X-ray observations. Count rate limit is in $0.3-6 \mathrm{keV}$ band and $L_{X}$ limit is in $0.5-6 \mathrm{keV}$ band.

implications for underluminous $\mathrm{SNe}$ and other unusual merger products. We conclude in Section 5.

\section{OBSERVATIONS}

\subsection{Optical Spectroscopy}

Eisenstein et al. (2006) identified 13 low-mass WD candidates with $\log g<7$. Kilic et al. (2010b) discuss followup observations of four of these targets. We selected 6 more targets for follow-up MMT spectroscopy. These targets are SDSS J002228.45+003115.5, J002207.65-101423.5, J123410.36-022802.8，J162542.10+363219.1，J204949.78+ 000547.3 and J225242.25-005626.6. We also observed SDSS J234536.48-010204.8, another ELM WD candidate identified by Liebert et al. (2004).

There are three more targets in the Eisenstein et al. (2006) ELM WD sample that lack follow-up spectroscopy. These targets (SDSS J1056+6536, J1426+0100, and J1630+4233) have $\log g>6.9$ based on the model fits by Eisenstein et al. (2006) and $\log g \approx 7$ based on the fits by Kilic et al. (2007a). Radial velocity observations of these objects will be useful for the identification of more short-period binary WD systems.

We used the $6.5 \mathrm{~m}$ MMT equipped with the Blue Channel Spectrograph to obtain moderate resolution spectroscopy of seven ELM WD candidates over 17 different nights between 2008 September and 2010 July. We operate the spectrograph with the 832 line $\mathrm{mm}^{-1}$ grating in second order, providing wavelength coverage $3600 \AA$ to $4500 \AA$ and a spectral resolution of 1.0-1.2 $\AA$, depending on the slit size used. We obtain all observations at the parallactic angle, with a comparison lamp exposure paired with every observation. We flux-calibrate using blue spectrophotometric standards (Massey et al. 1988), and we measure radial velocities using the cross-correlation package RVSAO (Kurtz \& Mink 1998). Our radial velocity measurement procedures are described in Kilic et al. (2009, 2010b).

\subsection{X-ray Observations}

\subsubsection{Motivation}

X-ray emission from radio MSPs is expected to be less highly beamed than radio emission, as a major component of the X-ray emission comes from the hot neutron star surface (Zavlin et al. 2002; Zavlin 2006). Blackbody emission from the surface of a possible pulsar companion to the ELM WDs will be gravitationally bent, allowing observation of $>75 \%$ of the neutron star surface in X-rays even if the radio pulsar beam (produced well above the surface) misses our line of sight (Beloborodov 2002). All 15 radio MSPs with precise positions in unconfused regions of the globular cluster 47 Tuc have been clearly detected in X-rays (Heinke et al. 2005; Bogdanov et al. 2006), with no observed correlation between their radio and $\mathrm{X}$-ray luminosities (expected, as they are produced via different mechanisms). There is also no evidence for differences in the X-ray properties of globular cluster versus Galactic MSPs (Bogdanov et al. 2006).

This result allows us to use the 47 Tuc MSP sample (with accurate $L_{X}$ values due to its well-known distance) to predict that other MSPs should have X-ray luminosities above $L_{X}(0.5-6 \mathrm{keV})=2 \times 10^{30} \mathrm{erg} \mathrm{s}^{-1}$, the minimum $L_{X}$ of MSPs in 47 Tuc. Thus, deep X-ray upper limits can rule out the presence of an MSP, as in the ELM WD SDSS J0917+4638 (Agüeros et al. 2009b). The MSPs in 47 Tuc show X-ray spectra dominated by thermal blackbody-like emission from the neutron star polar cap surfaces, with temperature $(1-3) \times 10^{6} \mathrm{~K}$ (Bogdanov et al. 2006). Some MSPs also show additional (sometimes much higher $L_{X}$ ) components due to magnetospheric emission or pulsar wind shocks, but there is no evidence for a MSP lacking the thermal component.

We obtained Chandra observations of three ELM WDs (SDSS J105353.89+520031.0, J123410.36-022802.8, and J092345.59+302805.0) to search for MSP X-ray emission. None of these WDs had been previously observed in the X-rays since the ROSAT All-Sky Survey (Voges et al. 1999), where the short exposure time was insufficient to place useful limits.

\subsubsection{Data Analysis}

We observed the three systems with Chandra's ACIS-S instrument in very faint mode, for the exposure times noted in Table 1. We used CIAO 4.2 (with CALDB 4.2.1) ${ }^{7}$ to reprocess the observations with current calibrations and reduce the backgrounds using very faint mode cleaning. We constructed images in the $0.3-6 \mathrm{keV}$ band and found no X-ray photons within the $1^{\prime \prime}$ error circles around each source. We use Poisson statistics (Gehrels 1986) to place 99\% confidence upper limits on the count rate. We compute distances to the ELM WDs using the models of Panei et al. (2007) and the SDSS $g$ magnitudes, and $N_{\mathrm{H}}$ values using the Colden tool ${ }^{8}$ (Dickey \& Lockman 1990). We use PIMMS ${ }^{9}$ and the X-ray spectrum of the faintest MSP in 47 Tuc (47 Tuc-T, $134 \mathrm{eV}$ blackbody) to produce $0.5-6 \mathrm{keV} L_{X}$ upper limits, which we list in Table 1.

The $99 \%$ confidence $L_{X}$ upper limits are all at least a factor of 10 fainter than the faintest MSP identified in 47 Tuc, and 21 times fainter than the median $L_{X}$ of these MSPs (Bogdanov et al. 2006). Thus the lack of X-ray emission from these WDs is strong evidence that the unseen companions are not MSPs.

\section{RESULTS}

Four of our seven targets exhibit significant radial velocity variations, with peak-to-peak velocity amplitudes between 124

\footnotetext{
7 http://cxc.harvard.edu/ciao/

8 http://asc.harvard.edu/toolkit/colden.jsp

9 http://asc.harvard.edu/toolkit/pimms.jsp
} 
Table 2

Orbital Parameters

\begin{tabular}{cccccc}
\hline \hline Object & $\begin{array}{c}P \\
(\text { days })\end{array}$ & $\begin{array}{c}K \\
\left(\mathrm{~km} \mathrm{~s}^{-1}\right)\end{array}$ & $\begin{array}{c}V_{\text {systemic }} \\
\left(\mathrm{km} \mathrm{s}^{-1}\right)\end{array}$ & $\begin{array}{c}\text { Spec. Conjunction } \\
(\mathrm{HJD}-2454000)\end{array}$ & $\begin{array}{c}\text { Mass Function } \\
\left(M_{\odot}\right)\end{array}$ \\
\hline J0022+0031 & $0.4914 \pm 0.0254$ & $80.8 \pm 1.3$ & $-20.3 \pm 0.8$ & 732.71818 & $0.02681 \pm 0.00190$ \\
J0022-1014 & $0.0799 \pm 0.0030$ & $145.6 \pm 5.6$ & $-38.5 \pm 3.7$ & 732.76530 & $0.02553 \pm 0.00310$ \\
J1234-0228 & $0.0914 \pm 0.0040$ & $94.0 \pm 2.3$ & $+50.3 \pm 1.8$ & 917.75366 & $0.00787 \pm 0.00067$ \\
J1625+3632 & $0.2324 \pm 0.0396$ & $58.4 \pm 2.7$ & $-95.0 \pm 2.1$ & 922.74700 & $0.00480 \pm 0.00105$ \\
J2252-0056 & $\ldots$ & $\ldots$ & $-23.2 \pm 0.8$ & $\ldots$ & $\ldots$ \\
J2345-0102 & $\cdots$ & $\ldots$ & $-161.2 \pm 0.4$ & $\ldots$ & $\cdots$ \\
\hline
\end{tabular}

Note. $\mathrm{J} 0022+0031$ has a period alias at $7.9 \mathrm{hr}$.

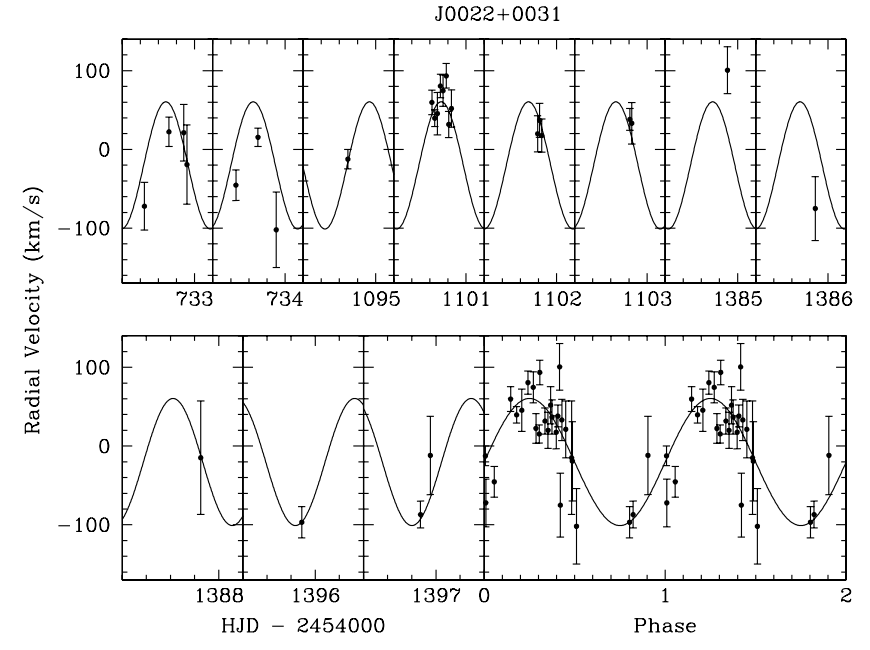

Figure 1. Radial velocities and the best-fit orbit for J0022+0031. The bottom right panel shows all of the data points phased with the best-fit period.

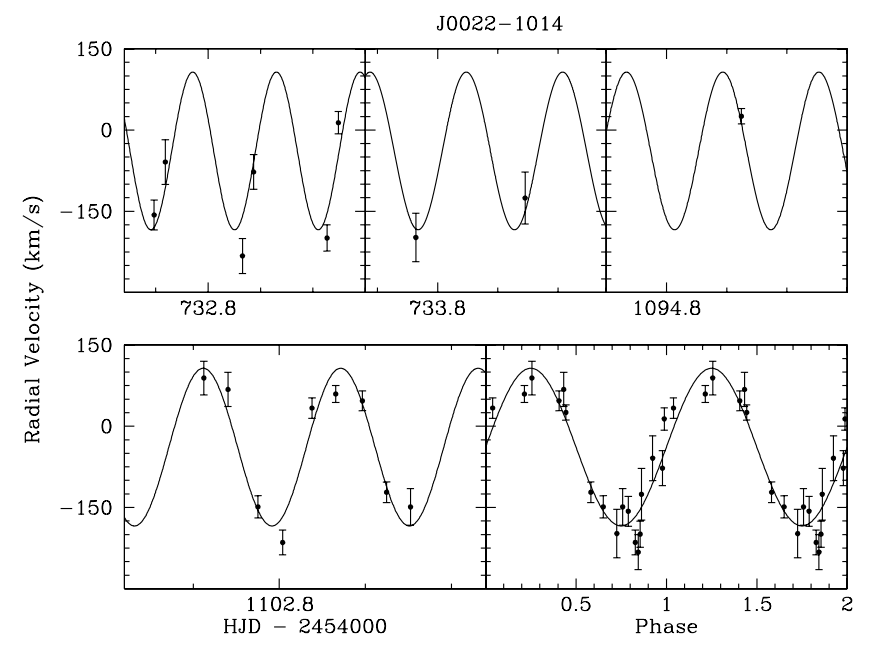

Figure 2. Same as Figure 1 but for J0022-1014.

and $232 \mathrm{~km} \mathrm{~s}^{-1}$. We weight each velocity measurement by its associated error and solve for the best-fit orbit using the code of Kenyon \& Garcia (1986). Figures 1-6 show the observed radial velocities and the best-fit orbits for our targets. The heliocentric radial velocities are best fit with circular orbits with periods 1.9-11.8 hr. We present the best-fit orbital period $(P)$, semiamplitude $(K)$ of the radial velocity variations, systemic velocity (which includes a small gravitational redshift term), the time of spectroscopic conjunction, and the mass function in Table 2. $\mathrm{J} 2049+0005$ is excluded from this list due to its low surface gravity, which implies that it is not a WD (see below).

Two of the stars in our sample, J2252-0056 and J2345-0102, do not show significant velocity variations. The

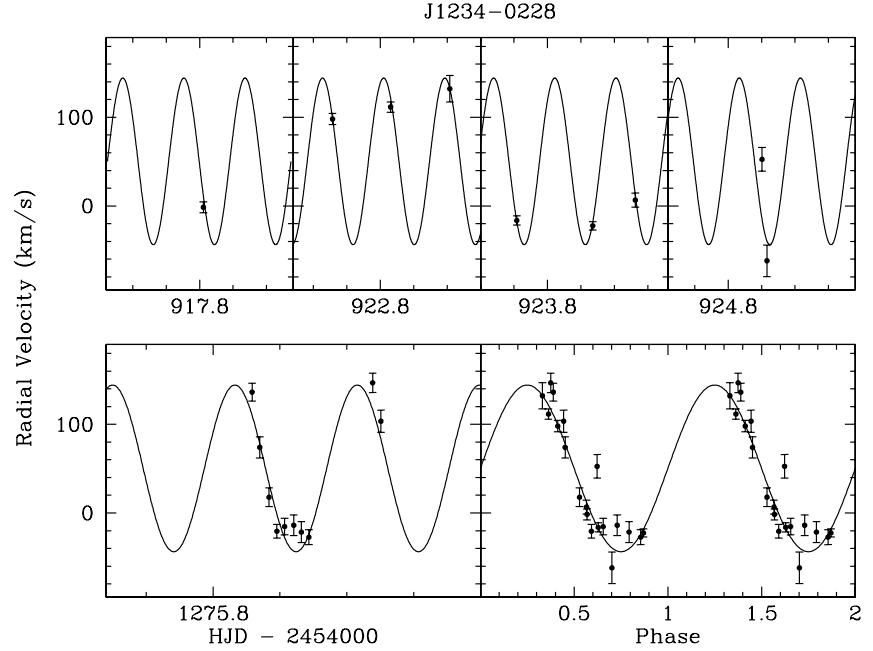

Figure 3. Same as Figure 1, but for J1234-0228.

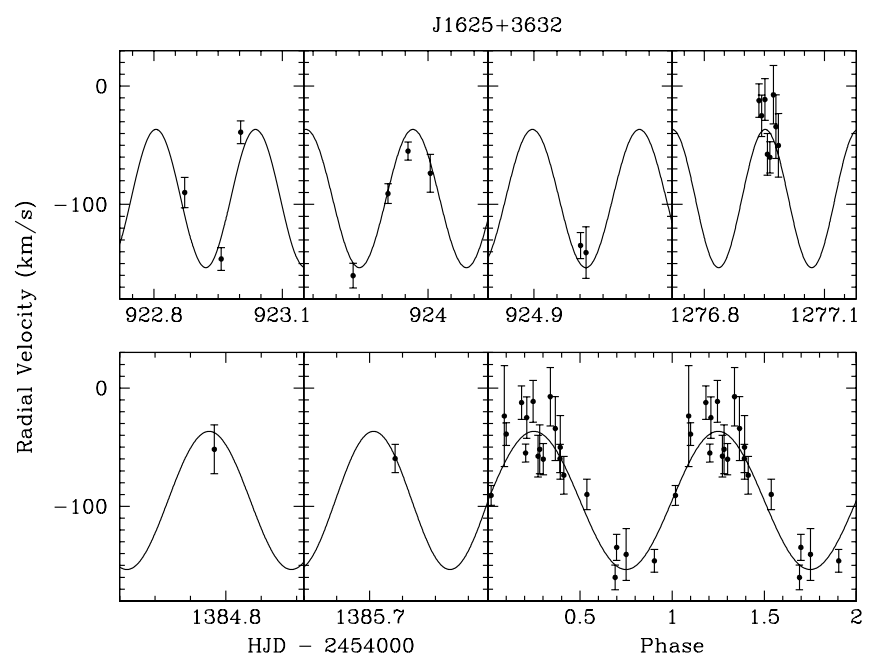

Figure 4. Same as Figure 1, but for J1625+3632.

best-fit orbital solutions have semi-amplitudes $25-43 \mathrm{~km} \mathrm{~s}^{-1}$. These two objects may be low-inclination systems or just single stars.

We perform model fits to each individual spectrum and also to the average composite spectra using synthetic DA WD spectra kindly provided by D. Koester. This model grid covers temperatures up to $30,000 \mathrm{~K}$. We use an NLTE grid computed by I. Hubeny (see Allende Prieto et al. 2009) to fit the spectrum of J2345-0102, which requires a solution slightly above $30,000 \mathrm{~K}$. We use the individual spectra to obtain a robust estimate of the errors in our analysis. Figure 7 shows the composite spectra and our fits using the entire wavelength range. The best-fit $T_{\text {eff }}$ and 


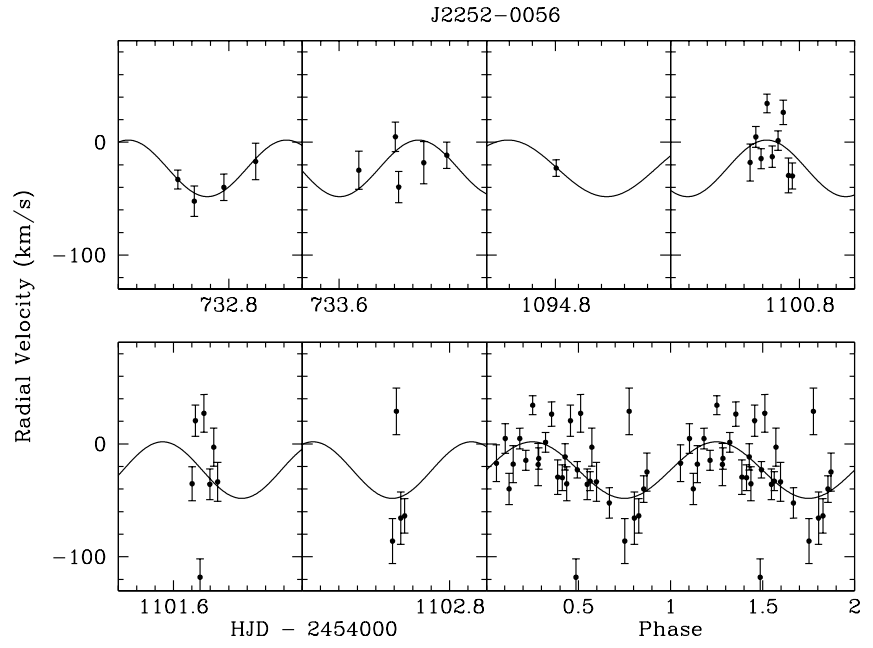

Figure 5. Same as Figure 1, but for J2252-0056.

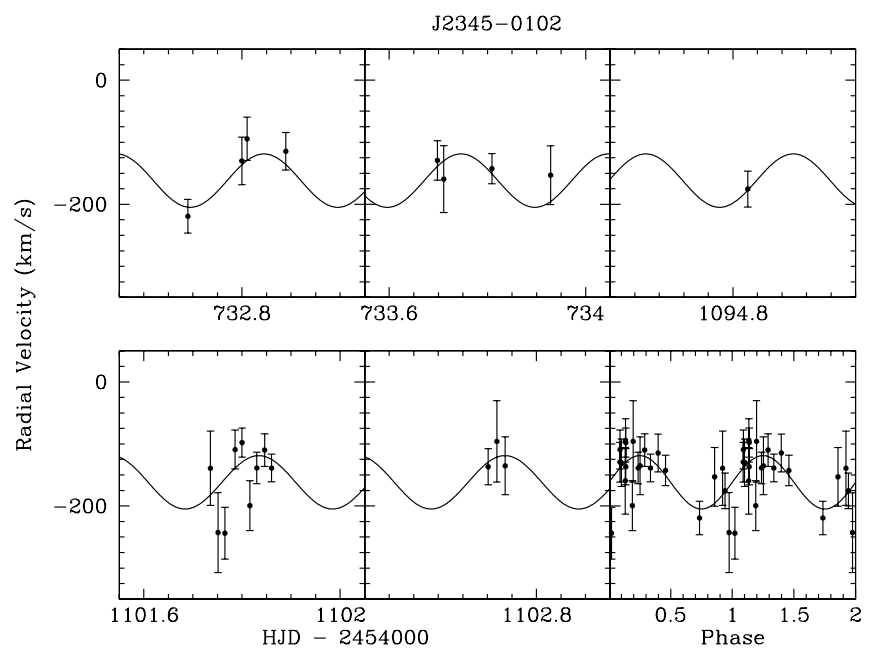

Figure 6. Same as Figure 1, but for J2345-0102.

$\log g$ values are given in Table 3. We obtain best-fit solutions of $17,890-33,130 \mathrm{~K}$ and $\log g=6.12-7.38$ for six of our targets, confirming that they are low-mass WDs.

Figure 8 shows the effective temperatures and surface gravities for our targets (red circles) plus the previously identified ELM WDs in the literature (Kilic et al. 2007b, 2009; Vennes et al. 2009; Kilic et al. 2010b; Brown et al. 2010a). Filled triangles show the WD companions to PSR J1012+5307 and J1911-5958A (van Kerkwijk et al. 1996; Bassa et al. 2006; see Section 4.4). Solid lines show the constant mass tracks for low mass WDs from Panei et al. (2007) models (updated by Kilic et al. 2010b). This figure shows that our six WD targets have masses ranging from 0.20 to $0.42 M_{\odot}$.

\subsection{Notes on Individual Objects}

\subsection{1. $J 0022+0031$}

The best-fit orbital period for J0022+0031 is $11.8 \mathrm{hr}$. However, the limited number of follow-up observations allow for an alias at $7.9 \mathrm{hr}$ period. $\mathrm{J} 0022+0031$ is a binary system containing a 120 Myr old $0.38 M_{\odot}$ WD and a $M \geqslant 0.21 M_{\odot}$ companion star. Based on the updated Panei et al. (2007) models, it has an absolute magnitude of $M_{\mathrm{g}}=9.8 \mathrm{mag}$ and a distance of 790 pc.

For all six WDs in our sample, we combine the spectra near maximum blueshifted and redshifted velocities into two

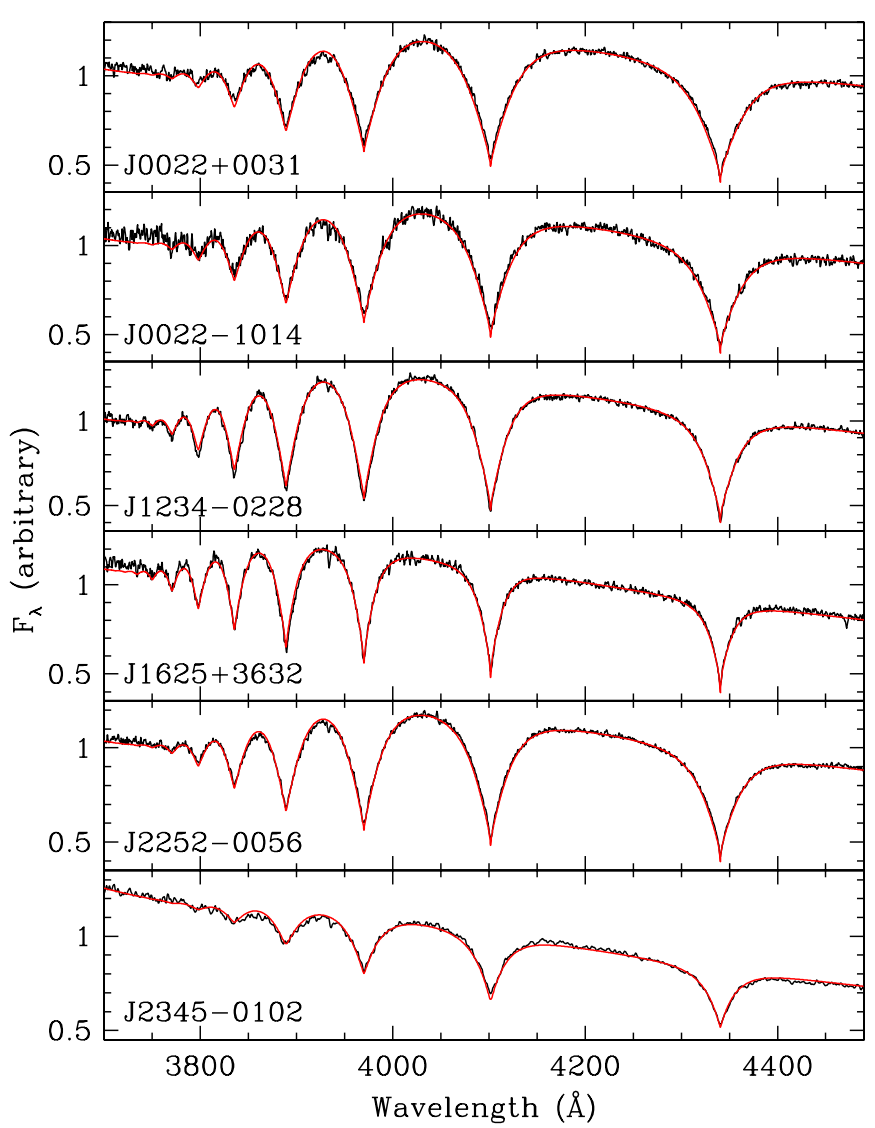

Figure 7. Model fits (red solid lines) to the composite spectra of our targets (jagged lines).

(A color version of this figure is available in the online journal.)

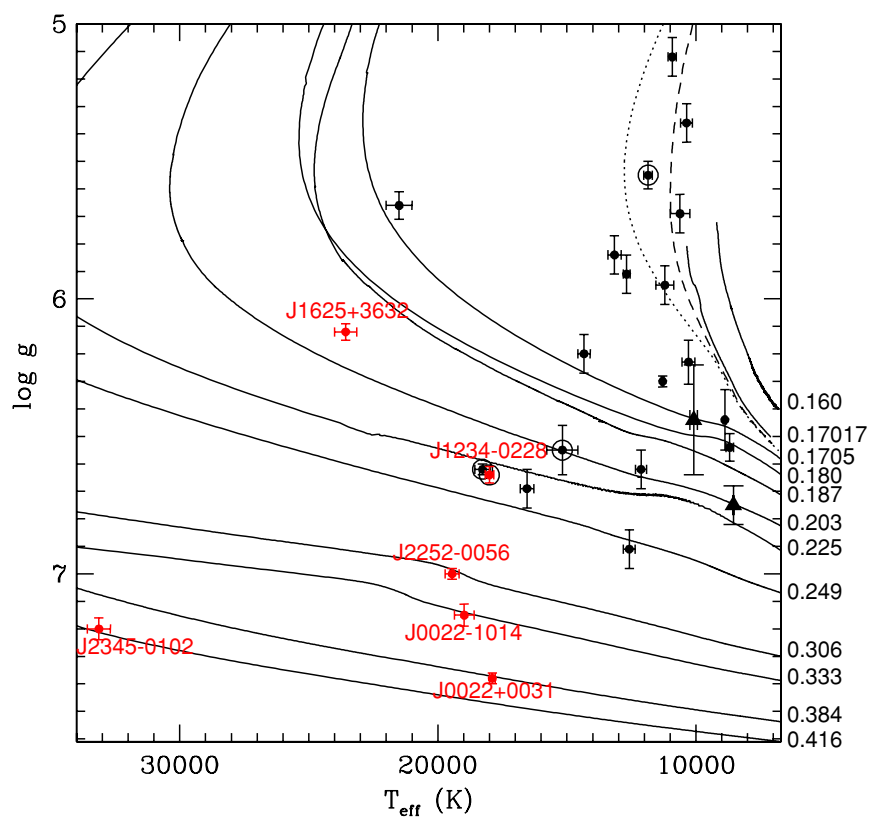

Figure 8. Best-fit solutions for the surface gravity and temperatures of our targets (red filled circles), overlaid on tracks of constant mass from Panei et al. (2007) models updated by Kilic et al. (2010a). The dashed and dotted lines show solar metallicity and halo metallicity $(Z=0.001)$ models of Serenelli et al. $(2001,2002)$ for $0.17 M_{\odot}$ WDs, respectively. Spectroscopically confirmed WDs from the ELM survey and the sdB star HD 188112 (Heber et al. 2003) are shown as black circles. Companions to millisecond pulsars PSR J1012+5307 and J1911-5958A are shown as triangles. The four objects with X-ray nondetections (Table 1 and Agüeros et al. 2009b) are marked by open circles.

(A color version of this figure is available in the online journal.) 
Table 3

Physical Parameters

\begin{tabular}{|c|c|c|c|c|c|c|c|c|}
\hline Object & $\begin{array}{l}T_{\text {eff }} \\
(\mathrm{K}) \\
\end{array}$ & $\log g$ & $\begin{array}{l}\text { Mass } \\
\left(M_{\odot}\right) \\
\end{array}$ & $\begin{array}{c}M_{2} \\
\left(M_{\odot}\right) \\
\end{array}$ & $\begin{array}{c}M_{2}\left(i=60^{\circ}\right) \\
\left(M_{\odot}\right)\end{array}$ & $\begin{array}{c}\text { NS } \\
\text { Prob. }\end{array}$ & $\begin{array}{l}\text { SN Ia } \\
\text { Prob. }\end{array}$ & $\begin{array}{l}\tau_{\text {merge }} \\
(\mathrm{Gyr}) \\
\end{array}$ \\
\hline $\mathrm{J} 0022+0031$ & $17890 \pm 110$ & $7.38 \pm 0.02$ & 0.38 & $\geqslant 0.21$ & 0.26 & $2.5 \%$ & $1.9 \%$ & $\leqslant 75.7$ \\
\hline J0022-1014 & $18980 \pm 380$ & $7.15 \pm 0.04$ & 0.33 & $\geqslant 0.19$ & 0.23 & $2.3 \%$ & $1.4 \%$ & $\leqslant 0.73$ \\
\hline $\mathrm{J} 1234-0228$ & $18000 \pm 170$ & $6.64 \pm 0.03$ & 0.23 & $\geqslant 0.09$ & 0.11 & $0.9 \%$ & $0.3 \%$ & $\leqslant 2.69$ \\
\hline $\mathrm{J} 1625+3632$ & $23570 \pm 440$ & $6.12 \pm 0.03$ & 0.20 & $\geqslant 0.07$ & 0.08 & $0.6 \%$ & $0.2 \%$ & $\leqslant 45.2$ \\
\hline $\mathrm{J} 2252-0056$ & $19450 \pm 270$ & $7.00 \pm 0.02$ & 0.31 & $\ldots$ & $\ldots$ & $\ldots$ & $\ldots$ & $\ldots$ \\
\hline $\mathrm{J} 2345-0102$ & $33130 \pm 450$ & $7.20 \pm 0.04$ & 0.42 & $\ldots$ & $\ldots$ & $\ldots$ & $\ldots$ & $\ldots$ \\
\hline
\end{tabular}

composite spectra. If there is a contribution from a companion object, it may be visible as an asymmetry in the line profiles. We do not see any obvious asymmetries in the line profiles and our optical spectroscopy does not reveal any spectral features from companion objects. A $M \geqslant 0.21 M_{\odot}$ main-sequence star companion to J0022+0031 would have $M_{I} \leqslant 10.0$ mag (Kroupa $\&$ Tout 1997$)$, brighter than the low-mass WD $\left(M_{I} \approx 10.4 \mathrm{mag}\right)$ and detectable in the $I$ band. Such a main-sequence star companion is ruled out based on the SDSS photometry.

Using the mean inclination angle for a random stellar sample, $i=60^{\circ}$, the companion has a mass of $0.26 M_{\odot}$. There is a $2.5 \%$ probability that the companion is a $1.4-3 M_{\odot}$ neutron star. Given the small probability of a neutron star companion and the unsuccessful searches for neutron stars around other ELM WDs (Section 2.2; see also Agüeros et al. 2009a, 2009b), the companion is most likely another WD. Short period binaries may lose angular momentum through gravitational wave radiation and merge within a Hubble time. The merger time for the J0022+0031 system is longer than a Hubble time.

\subsubsection{J0022-1014}

$\mathrm{J} 0022-1014$ is a $1.9 \mathrm{hr}$ period binary system with a $0.33 M_{\odot}$ WD primary and a $M \geqslant 0.19 M_{\odot}$ secondary. The primary WD is $70 \mathrm{Myr}$ old, it has $M_{\mathrm{g}}=9.2 \mathrm{mag}$, and is located at a distance of $1.3 \mathrm{kpc}$. A $M \geqslant 0.19 M_{\odot}$ main-sequence star companion would have $M_{I} \leqslant 10.3 \mathrm{mag}, 30 \%$ fainter than the ELM WD. Such a companion is ruled out based on the SDSS photometry. The companion to J0022-1014 is most likely another WD, and specifically a low-mass WD. There is a $83 \%$ probability that the companion is less massive than $0.45 M_{\odot}$, a He-core low-mass WD. This system will merge in less than $730 \mathrm{Myr}$.

J0022-1014 has a proper motion of $\mu_{\alpha} \cos \delta=-7.8$ and $\mu_{\delta}=-13.2$ mas $\mathrm{yr}^{-1}$ (Munn et al. 2004). Based on the mass and radius estimates, the gravitational redshift of the WD is $8.1 \mathrm{~km} \mathrm{~s}^{-1}$. Therefore, the true systemic velocity is $-46.6 \mathrm{~km} \mathrm{~s}^{-1}$. The velocity components with respect to the local standard of rest as defined by Hogg et al. (2005) are $U=95 \pm 23, V=-53 \pm 21$, and $W=33 \pm 8 \mathrm{~km} \mathrm{~s}^{-1}$. $\mathrm{J} 0022-1014$ is a disk star.

\subsection{3. $J 1234-0228$}

J1234-0228 was originally identified as a very low mass WD by Liebert et al. (2004) and is included in the DR4 WD catalog of Eisenstein et al. (2006). Based on our MMT observations over five different nights separated by about a year, J1234-0228 is a binary system with an orbital period of $2.2 \mathrm{hr}$. The visible component of the binary is a 70 Myr old WD with $M_{\mathrm{g}}=8.4 \mathrm{mag}$ at a distance of $780 \mathrm{pc}$. For an inclination angle of $60^{\circ}$, the mass function requires a $0.11 M_{\odot}$ companion. A main-sequence companion of this mass would contribute $7 \%$ excess flux in the $I$ band. No excess flux is observed in the SDSS photometry and spectrum of this object, therefore the companion is most likely another WD (see also Liebert et al. 2004).

There is a $94 \%$ chance that the companion is a low-mass $\left(<0.45 M_{\odot}\right) \mathrm{WD}$, and more interestingly there is a $85 \%$ probability that the combined mass of the two stars in this system is lower than $0.45 M_{\odot}$. The merger time for the J1234-0228 system is shorter than 2.7 Gyr. Therefore, within the next few Gyr, this system will merge and likely form a single low-mass WD.

J1234-0228 has a proper motion of $\mu_{\alpha} \cos \delta=-14.5$ and $\mu_{\delta}=-12.3$ mas $\mathrm{yr}^{-1}$ (Munn et al. 2004). Based on the mass and radius estimates, the gravitational redshift of the $\mathrm{WD}$ is $3.7 \mathrm{~km} \mathrm{~s}^{-1}$. Therefore, the true systemic velocity is $+46.6 \mathrm{~km} \mathrm{~s}^{-1}$. The velocity components with respect to the local standard of rest as defined by Hogg et al. (2005) are $U=-5 \pm 14, V=-78 \pm 12$, and $W=21 \pm 7 \mathrm{~km} \mathrm{~s}^{-1}$. $\mathrm{J} 1234-0228$ is also a disk star.

\subsection{4. $J 1625+3632$}

$\mathrm{J} 1625+3632$ has a best-fit orbital period of $5.6 \mathrm{hr}$. The MMT spectrum reveals a weak He I line at $4471 \AA$. Our best-fit DA WD model has $T_{\text {eff }}=23570 \mathrm{~K}$ and $\log g=6.12$, similar to the sdB star HD 188112 (Heber et al. 2003). Based on these model fits, the primary is a $\approx 160$ Myr old $0.20 M_{\odot}$ WD at a distance of $2.8 \mathrm{kpc}$. J1625+3632 does not display a significant proper motion, but given its distance and systemic velocity, it is likely a member of the thick disk or halo.

The relatively small semi-amplitude $\left(K=58.4 \mathrm{~km} \mathrm{~s}^{-1}\right)$ of the radial velocity variations imply that the lower limit on the mass of the companion is $0.07 M_{\odot}$. A main-sequence companion of this mass would contribute less than $1 \%$ in the optical and would not be detected in our observations or the SDSS photometry. Given the accuracy of the SDSS photometry, any main-sequence companion more massive than about $0.17 M_{\odot}$ would have been detected. Hence, only $0.07-0.17 M_{\odot}$ mainsequence companions are allowed based on the mass function and optical photometry. Near-infrared observations will be useful to rule out such companions.

$\mathrm{J} 1625+3632$ may be a low inclination system. However, the probability of a neutron star companion is only $0.6 \%$. Given the narrow range of possible main-sequence companions and the unlikelihood of a neutron star companion, the companion is likely another WD. There is a $96 \%$ chance that the companion is less massive than $0.45 M_{\odot}$, i.e., a low-mass He-core WD.

\subsection{5. $J 2049+0005$}

Figure 9 shows the best-fit WD model to our MMT spectrum of J2049+0005. This star was identified as a $T_{\text {eff }}=8660 \mathrm{~K}$ and $\log g=5.48$ WD by Eisenstein et al. (2006). Further analysis of the same SDSS spectrum by Kilic et al. (2007a) suggested that $\mathrm{J} 2049+0005$ has $\log g<5$, and therefore is not a WD. Similarly, 


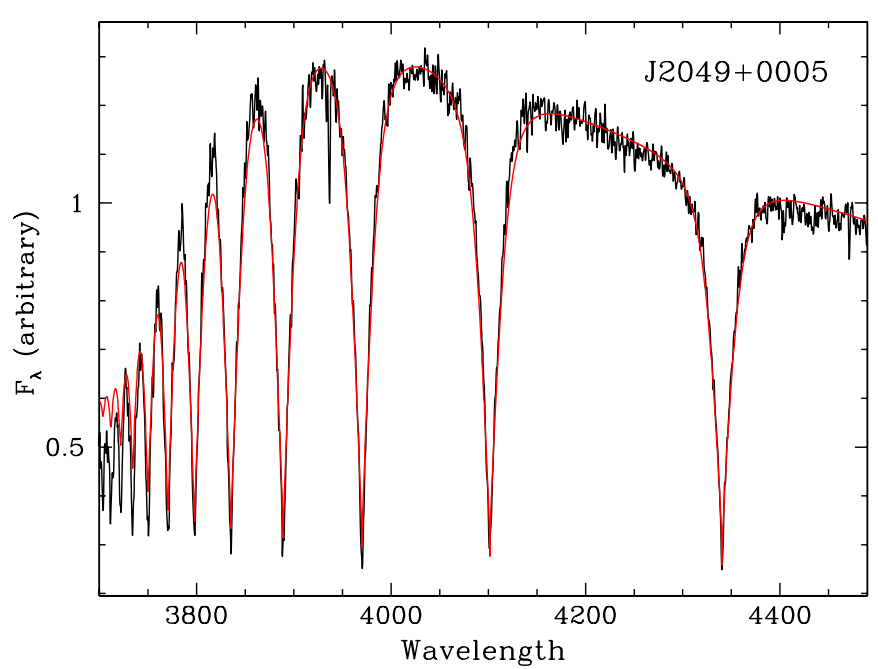

Figure 9. Best-fit model (red solid line) to the observed spectrum of SDSS $\mathrm{J} 2049+0005$. The best-fit model has $\log g=5$ and does not match the higher order Balmer lines. J2049+0005 is likely an A star.

(A color version of this figure is available in the online journal.)

our model fits to the higher resolution and higher signal-to-noise ratio MMT spectrum find a best-fit solution that is exactly our lowest gravity model $(\log g=5)$. This model poorly matches the high-order Balmer lines, indicating that the surface gravity is lower than $\log g=5$ and that $\mathbf{J} 2049+0005$ is most likely an A star.

\subsubsection{J2252-0056}

Based on 30 different spectra, the best-fit orbital period is $10.3 \mathrm{hr}$ with $K=25.1 \pm 1.5 \mathrm{~km} \mathrm{~s}^{-1}$. However, the radial velocity variations are not significant. The best-fit orbit has a $\chi^{2}$ slightly better than a constant-velocity fit. If J2252-0056 is a binary, it is likely a low inclination or a pole-on system. Given the $25 \mathrm{~km} \mathrm{~s}^{-1}$ upper limit to the velocity semi-amplitude, J2252-0056 could be a binary with either month-long (assuming an $0.5 M_{\odot}$ companion and $i=60^{\circ}$ ) orbital period or relatively pole-on inclination $\left(M \sin (i)=0.03-0.05 M_{\odot}\right.$ for $2-12 \mathrm{hr}$ periods $)$. There is no evidence of a companion in our MMT spectrum or the SDSS observations.

\subsubsection{J2345-0102}

J2345-0102 is another object for which we detect no significant velocity variations. The best-fit orbital period is $7.2 \mathrm{hr}$ with $K=43.0 \pm 0.7 \mathrm{~km} \mathrm{~s}^{-1}$, but the best-fit orbit is a slightly better fit to the data than a constant-velocity fit. Again, if J2345-0102 is a binary, it may be a low inclination system. The observed $43 \mathrm{~km} \mathrm{~s}^{-1}$ upper limit to the velocity semi-amplitude corresponds to a binary with either month-long orbital period or relatively pole-on inclination $\left(M \sin (i)=0.05-0.10 M_{\odot}\right.$ for 2-12 hr periods).

The MMT spectrum of this object is best explained with a $23 \mathrm{Myr}$ old $0.42 M_{\odot} \mathrm{WD}$ at a distance of $2.0 \mathrm{kpc}$. Based on 5 epochs from the USNO-B+SDSS, Munn et al. (2004) measure a proper motion of $\mu_{\alpha} \cos \delta=-26.0$ and $\mu_{\delta}=$ $-36.6 \mathrm{mas} \mathrm{yr}^{-1}$. Given its relatively large systemic velocity, proper motion, and distance, J2345-0102 is clearly a halo star. About half of the known $0.4 M_{\odot}$ WDs seem to be single (see the discussion in Kilic et al. 2007c). Hence, the lack of significant radial velocity variations is not very surprising for this system.

\section{DISCUSSION}

\subsection{The Binary Fraction of ELM WDs}

Kilic et al. (2007b, 2010b), Brown et al. (2010a), and this paper discuss radial velocity observations of 11 ELM WD candidates identified in the SDSS DR4 (Eisenstein et al. 2006) and 12 ELM WDs identified in the Hypervelocity Star Survey of Brown et al. (2006). One of the targets is common to both samples. In addition, one of the DR4 targets $(\mathrm{J} 2049+0005)$ is most likely an A-star. Kilic et al. (2009, 2010c), Kawka et al. (2010), and Steinfadt et al. (2010) describe radial velocity observations of two more ELM WDs identified in the literature. Thus, there are 23 (candidate) ELM WD systems with comprehensive radial velocity measurements. All but three of these systems (J1448+1342, J2252-0056, and J2345-0102) are in binary systems.

Limiting this sample to 19 WDs with $M \leqslant 0.25 M_{\odot}, 18$ are in short period binary systems. Thus, close binary evolution is required for the formation of ELM WDs. The only system that does not display significant radial velocity variations, $\mathrm{J} 1448+1342$, is at the upper mass limit of this selection; all 18 WDs with $M<0.25 M_{\odot}$ are indeed binaries. The average velocity semi-amplitude of these 18 binaries is $236 \mathrm{~km} \mathrm{~s}^{-1}$, whereas the upper limit for the velocity semi-amplitude of $\mathrm{J} 1448+1342$ is $35 \mathrm{~km} \mathrm{~s}^{-1}$. An average system viewed at $i \leqslant 8.5$ would be consistent with the observations of J1448+1342. For a randomly distributed sample of orbital inclinations, there is a $1.1 \%$ chance that $i \leqslant 8.5$. Thus, there is a $21 \%$ likelihood of finding one of the 19 systems with $i \leqslant 8.5$. It is possible that $\mathrm{J} 1448+1342$ is a pole-on binary system (see also Brown et al. 2010a). Hence, the binary fraction of $M \leqslant 0.25 M_{\odot}$ WDs is at least $95 \%$ and it may be as high as $100 \%$.

This result is in contrast to the binary fraction of slightly more massive WDs. Maxted et al. (2000), Napiwotzki et al. (2004), and Brown et al. (2010) demonstrate that about 30\%-50\% of $M \approx 0.4 M_{\odot}$ WDs are single (see also Kilic et al. 2010a). Single low-mass WDs may form through enhanced mass loss from a metal-rich progenitor star (Kilic et al. 2007c), but we do not expect even the most metal-rich stars to loose enough mass to create $\sim 0.2 M_{\odot}$ WDs. Here we confirm this expectation that the lowest mass WDs all form in close binary systems.

\subsection{A Dozen Merger Systems}

Double degenerate (DD) merger systems are one of the two main accepted formation channels for Type Ia supernovae (Iben \& Tutukov 1984; Branch et al. 1995; Kotak 2008; Di Stefano 2010, and references therein). However, only a handful of DD systems are known to have combined mass above the Chandrasekhar limit and short enough orbital periods to merge through gravitational wave radiation within a Hubble time. Napiwotzki et al. (2001) started the heroic SPY survey for short period DD systems. They used the Very Large Telescope to observe 1014 stars brighter than $16.5 \mathrm{mag}$, including $75 \%$ of the known WDs accessible from the southern hemisphere. Napiwotzki et al. (2004) report that the SPY survey discovered about 100 new DD systems, one of which is a potential SNe Ia progenitor (WD 2020-425; Napiwotzki et al. 2007). Geier et al. (2010) describe the discovery of another massive DD merger system and also a summary of the current discoveries from the SPY survey (their Figure 4).

Figure 10 shows the total masses and periods for the short period DD systems (including $\mathrm{sdB}+\mathrm{WD}$ ) discovered by the SPY survey (kindly made available to us by S. Geier) 
Table 4

Merger Systems in the ELM Survey

\begin{tabular}{lcccccccr}
\hline \hline Object & $\begin{array}{c}T_{\text {eff }} \\
(\mathrm{K})\end{array}$ & $\log g$ & $\begin{array}{c}P \\
(\text { days })\end{array}$ & $\begin{array}{c}K \\
\left(\mathrm{~km} \mathrm{~s}^{-1}\right)\end{array}$ & $\begin{array}{c}\text { Mass } \\
\left(M_{\odot}\right)\end{array}$ & $\begin{array}{c}M_{2} \\
\left(M_{\odot}\right)\end{array}$ & $\begin{array}{c}M_{2}\left(i=60^{\circ}\right) \\
\left(M_{\odot}\right)\end{array}$ & $\begin{array}{c}\tau_{\text {merge }} \\
(\mathrm{Gyr})\end{array}$ \\
\hline J0022-1014 & 18980 & 7.15 & 0.07989 & 145.6 & 0.33 & $\geqslant 0.19$ & 0.23 & $\leqslant 0.73$ \\
J0755+4906 & 13160 & 5.84 & 0.06302 & 438.0 & 0.17 & $\geqslant 0.81$ & 1.12 & $\leqslant 0.22$ \\
J0818+3536 & 10620 & 5.69 & 0.18315 & 170.0 & 0.17 & $\geqslant 0.26$ & 0.33 & $\leqslant 8.89$ \\
J0822+2753 & 8880 & 6.44 & 0.24400 & 271.1 & 0.17 & $\geqslant 0.76$ & 1.05 & $\leqslant 8.42$ \\
J0849+0445 & 10290 & 6.23 & 0.07870 & 366.9 & 0.17 & $\geqslant 0.64$ & 0.88 & $\leqslant 0.47$ \\
J0923+3028 & 18350 & 6.63 & 0.04495 & 296.0 & 0.23 & $\geqslant 0.34$ & 0.44 & $\leqslant 0.13$ \\
J1053+5200 & 15180 & 6.55 & 0.04256 & 264.0 & 0.20 & $\geqslant 0.26$ & 0.33 & $\leqslant 0.16$ \\
J1233+1602 & 10920 & 5.12 & 0.15090 & 336.0 & 0.17 & $\geqslant 0.86$ & 1.20 & $\leqslant 2.14$ \\
J1234-0228 & 18000 & 6.64 & 0.09143 & 94.0 & 0.23 & $\geqslant 0.09$ & 0.11 & $\leqslant 2.69$ \\
J1436+5010 & 16550 & 6.69 & 0.04580 & 347.4 & 0.24 & $\geqslant 0.46$ & 0.60 & $\leqslant 0.10$ \\
J2119-0018 & 10360 & 5.36 & 0.08677 & 383.0 & 0.17 & $\geqslant 0.75$ & 1.04 & $\leqslant 0.54$ \\
NLTT 11748 & 8690 & 6.54 & 0.23503 & 273.4 & 0.18 & 0.76 & $\ldots$ & 7.20 \\
\hline
\end{tabular}

Notes. This table is based on the data presented in Kilic et al. (2010b), Brown et al. (2010a), Kilic et al. (2010c), and this paper. See Mullally et al. (2009), Steinfadt et al. (2010), and Kawka et al. (2010) for additional observations of J1053+5200, J1436+5010, and NLTT 11748.

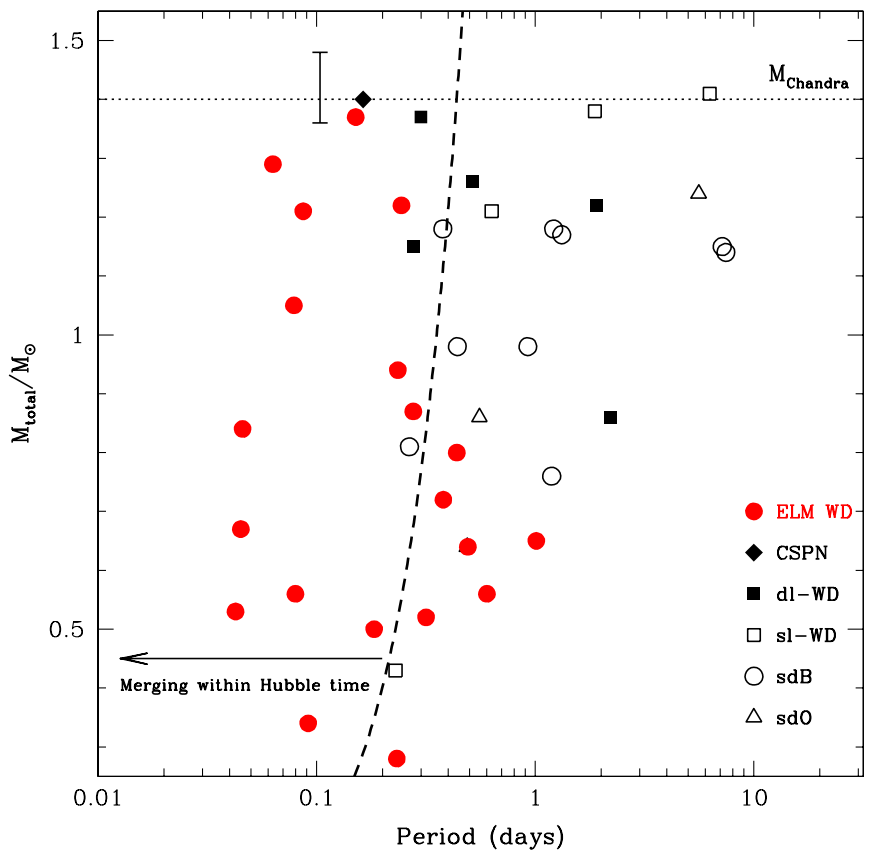

Figure 10. Total mass plotted against logarithmic period of double degenerate systems from the SPY survey (Geier et al. 2010) and our ELM WD sample (red filled circles). ELM WDs, central stars of planetary nebulae (CSPN), doublelined/single-lined WD systems, and sdB/sdO systems are shown with different symbols. Geier et al. (2010) assumes an inclination angle of $52^{\circ}$ for the singlelined systems, whereas we use an inclination angle of $60^{\circ}$ for our systems.

(A color version of this figure is available in the online journal.)

and our ELM WD sample. To explain the observed SNe Ia rate, only one out of 1000 WDs is expected to be a SNe Ia progenitor (Nelemans et al. 2001). Hence, the relatively few number of potential SNe Ia progenitors in the SPY survey is not surprising. What is striking is that the number of merger systems found in the SPY survey is significantly smaller than our ELM WD survey. We discovered 12 binary WD merger systems $\left(\tau_{\text {merge }}<\tau_{\text {hubble }}\right)$ in a sample of 23 stars, whereas the SPY survey discovered a handful of such systems in a sample of 1000 stars. The reason for this is the binary common envelope evolution required to form ELM WDs.

Figure 11 shows the total masses and periods for previously identified WD + WD binary systems in the literature (Nelemans

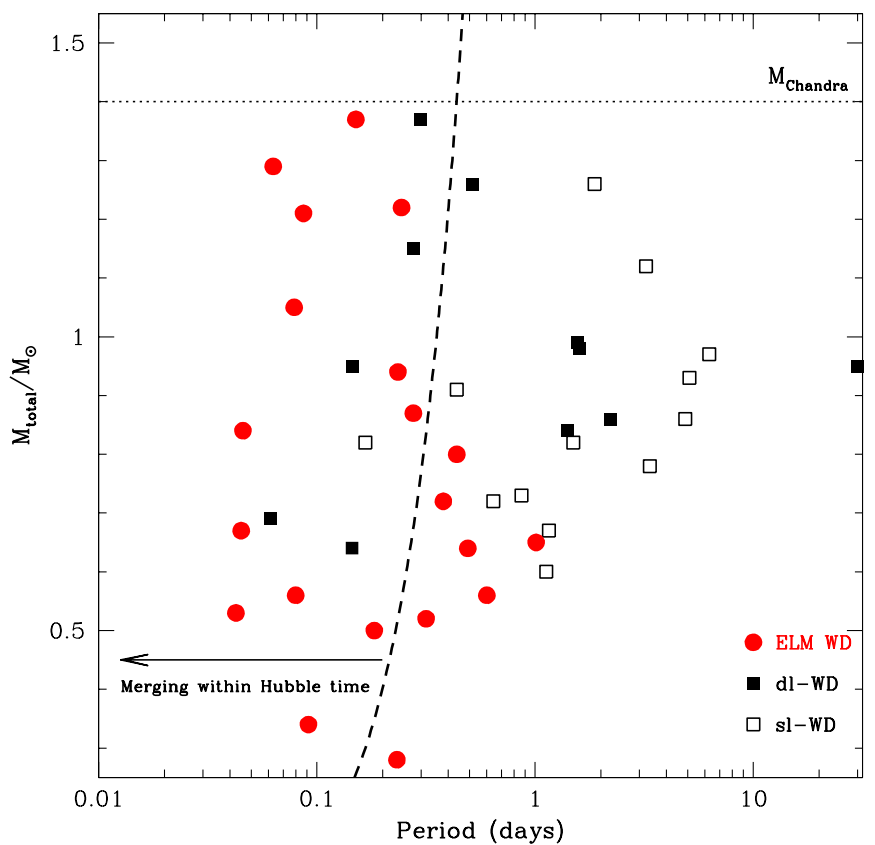

Figure 11. Same as Figure 10, but including only the double WD systems found in the literature (Nelemans et al. 2005; Napiwotzki et al. 2007).

(A color version of this figure is available in the online journal.)

et al. 2005; Napiwotzki et al. 2007). There were only six double WD merger systems known prior to our work. With the additional 12 merger systems presented in Table 4, we now have tripled the number of WD merger systems known.

\subsection{The Period Distribution of Binary WDs}

Nelemans et al. (2001) studied the period and mass distribution of double WD systems using population synthesis models. The details of these models including selection effects due to orbital evolution of the systems (disappearance of the shortest period systems due to mergers), target selection biases in the current surveys, and different cooling models are discussed in their paper. Nelemans et al. (2001) find a correlation between the masses and periods; more massive WD primaries have longer periods. This is not surprising; shorter period systems would start interacting earlier in their evolution compared to longer 

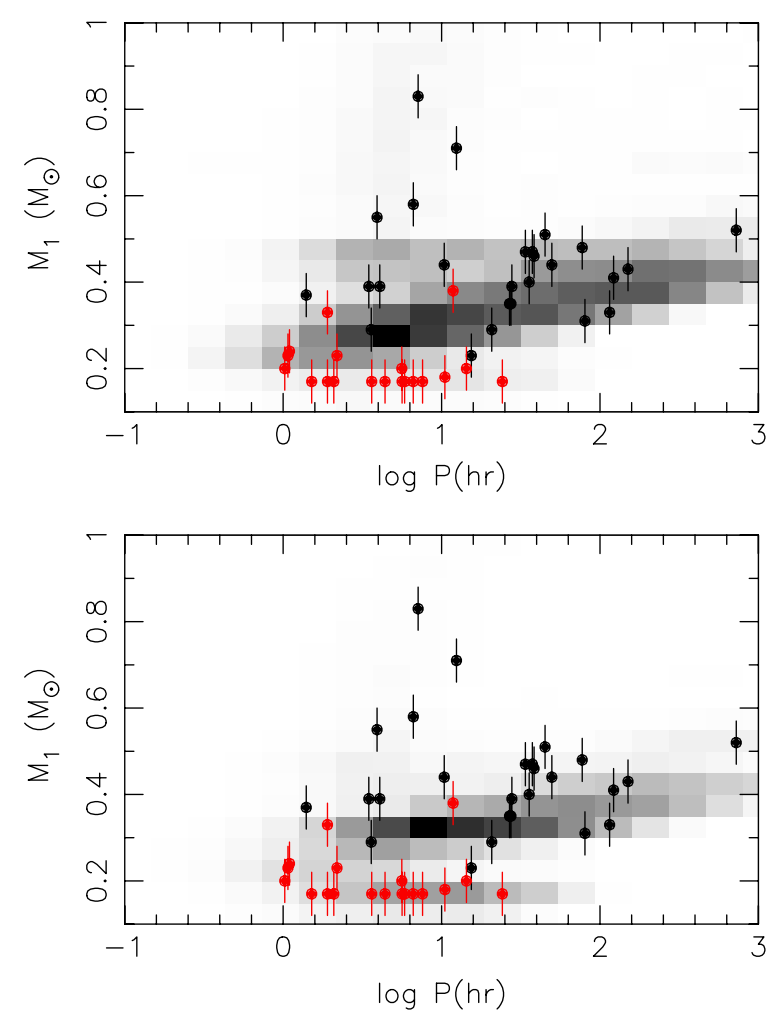

Figure 12. Model population of double WDs as a function of orbital period and mass of the brighter WD of the pair (kindly made available by G. Nelemans). The top panel uses Hansen (1999) cooling models whereas the bottom panel uses modified Driebe et al. (1998) cooling models. The observed binary WD population (Nelemans et al. 2005) and our ELM WD sample (red filled circles) are also shown.

(A color version of this figure is available in the online journal.)

period systems and experience enhanced mass loss during the red giant phase, hence end up as lower mass WDs. They also find that the mass ratio distribution peaks at $q=1$, favoring equal mass binary components. This distribution is consistent with the observed mass ratio distribution of binary WDs known prior to our work.

Figure 12 shows the masses and orbital periods for our sample of ELM WDs and the previously known binary WD systems in the literature compared to the population synthesis models of Nelemans et al. (2001, 2004, kindly made available to us by G. Nelemans). Depending on which cooling model is used, the model predictions change somewhat (see Nelemans et al. 2004, for a discussion of the cooling models used here). However, the main trend in the mass versus period distribution is evident; lower mass primaries are in shorter period systems. This result is consistent with the observed distribution of masses and periods for previously known binary WD systems and the ELM WD systems. All but one of the $M<0.3 M_{\odot}$ systems have periods shorter than a day, ${ }^{10}$ whereas the majority of the more massive WDs have periods longer than a day.

The overall number distribution of the population synthesis models and the observations cannot be directly compared without taking into account the obvious biases in the target selection. The sample of previously known binary WDs are selected from a magnitude limited sample down to 16-17 mag, whereas our sample mainly comes from fainter targets selected

10 The single velocity non-variable system, $\mathrm{J} 1448+1342$, is probably a pole-on or a relatively long-period binary system. from the SDSS, which has its own spectroscopic target selection bias. Nevertheless, an overabundance of $0.17 M_{\odot}$ WDs is evident in Figure 12. A comparison between the population synthesis models using Hansen (1999, top panel) and the modified Driebe et al. (1998, bottom panel) cooling models shows that the expected number of the lowest mass WDs changes depending on the cooling model used. The latter models predict a significantly larger number of $0.17 M_{\odot}$ WDs. Based on the Panei et al. (2007) models, a $0.25 M_{\odot}$ WD cools $4 \times$ faster than a $0.17 M_{\odot}$ WD down to an absolute magnitude of $M_{g}=10$ mag. Hence, the observed mass distribution of ELM WDs in a magnitude-limited survey is skewed toward lower mass objects (Brown et al. 2010b). Population synthesis models and a large, unbiased, magnitude-limited survey of low-mass and ELM WDs like that of Brown et al. (2010a) will be useful to constrain the ELM WD cooling models.

Another important prediction of the population synthesis models is that there should not be many systems with periods less than an hour. ${ }^{11}$ We have three ELM WD systems with periods close to $1 \mathrm{hr}$. The shortest period system, J1053+5200 has a period of $61 \mathrm{~min}$ (Kilic et al. 2010b; Mullally et al. 2009). The shortest period hydrogen-rich cataclysmic variable (CV) V485 Cen has an orbital period of 59 minutes (Augusteijn et al. 1996). Both short period binary WDs and CVs are expected to contribute to the AM CVn population, which contains interacting WD binary systems with orbital periods ranging from a few minutes to about an hour. The two longest period AM CVn systems have periods of $56 \mathrm{~min}$ and $66 \mathrm{~min}$, respectively (Roelofs et al. 2007). It seems that hydrogen-deficient AM $\mathrm{CVn}$ interacting binary systems have periods less than about an hour and the hydrogen-rich detached WD systems have orbital periods about an hour or longer.

\subsection{The Minimum Mass for Low-mass WDs}

Before the SDSS, ELM WDs were traditionally found as companions to MSPs. The masses for the MSP companions are usually measured from the pulsar timing measurements since the WDs are usually too faint for follow-up optical spectroscopy. van Kerkwijk et al. (1996) and Bassa et al. (2006) obtained Keck and VLT spectroscopy of companions to two MSP systems. van Kerkwijk et al. (1996) derive $T_{\text {eff }}=8550 \pm 25 \mathrm{~K}$ and $\log \mathrm{g}=$ $6.75 \pm 0.07$ for the companion to PSR J1012+5307, whereas Bassa et al. (2006) obtain $T_{\text {eff }}=10,090 \pm 150 \mathrm{~K}$ and $\log \mathrm{g}=$ $6.44 \pm 0.20$ for the companion to PSR J1911-5958A. These two WDs are included in Figure 8.

Kilic et al. (2007a, 2007b) discovered the lowest surface gravity WD known at the time, SDSS J0917+4638, with $\log g=5.55$. Brown et al. (2010a) discovered two WDs with even lower surface gravities, SDSS J1233+1602 and J2119-0018. These WDs are included in our ELM WD sample and in Figure 8. Based on the Serenelli et al. $(2001,2002)$ and Panei et al. (2007) models, all of these WDs, including the two MSP companions, have $M \geqslant 0.17 M_{\odot}$. There are about a dozen WDs in Figure 8 with $M \approx 0.17 M_{\odot}$, but none below that mass limit. However, this result is most likely due to observational biases present in the SDSS and the Hypervelocity Star Survey. The former is, of course, an incomplete spectroscopic survey and the latter has a color selection that excludes $T_{\text {eff }} \leqslant 10,000$ K objects with $\log g=5-6$ (see Figure 1 in Brown et al. 2010a). Based on the Panei et al. (2007) models, a $0.16 M_{\odot}$ WD takes

11 These systems either merge or evolve into AM CVn systems relatively quickly. 
15 Gyr to cool down from $9200 \mathrm{~K}$ to about $7000 \mathrm{~K}$ with an increase in surface gravity from $\log g=5.7$ to 6.4 and a decrease in luminosity from $M_{g}=8.1$ to $11.1 \mathrm{mag}$. Such WDs are not included in the Hypervelocity Star Survey color selection.

Based on radio timing measurements, 45 out of 99 MSPs with periods less than $20 \mathrm{~ms}$ have companions less massive than $0.17 M_{\odot}$ (B. Kiziltan 2010, private communication). The spectral types of the companions are unknown. However, a large fraction of these objects are expected to be low-mass WDs; nature produces $M<0.17 M_{\odot}$ WDs in MSP systems. Lower mass ELM WDs should be produced in binary WD systems as well. Population synthesis calculations for double WDs do not predict many WDs below $0.17 M_{\odot}$, but such WDs may eventually be found in double WD systems in the SDSS or other spectroscopic surveys.

\subsection{Merger Products: (Underluminous) SNe Ia, AM CVn, and RCrB Stars}

The end result of the future evolution of the 12 ELM WD merger systems in our sample depends on the mass ratios of the two components. The systems with extreme mass ratios $(q \leqslant 0.2)$ should have stable mass transfer at initial Roche lobe filling. Stable mass transfer leads to the formation of AM $\mathrm{CVn}$ systems that transfer angular momentum back to the orbit, which increases the orbital period. If the higher mass WDs in such systems have masses close to the Chandrasekhar limit, accretion may result in a Type Ia SNe explosion. However, the probability of such an event is small $(\leqslant 7 \%)$ for our systems. This probability would be higher if sub-Chandrasekhar mass WD explosions also contribute to the observed Type Ia SNe population (Sim et al. 2010; Kromer et al. 2010, and references therein). However, our ELM survey is biased against $\mathrm{SNe}$ Ia progenitors due to the selection of very low-mass primaries.

Figure 13 shows the mass ratios and periods for our ELM WD sample plus the previously known binary WD systems. Single-lined systems are shown as filled triangles, whereas the double-lined systems and the eclipsing WD system NLTT 11748 are shown as filled circles. Open circles mark the objects with merger times shorter than a Hubble time. Out of the 18 merger systems, six have mass ratios smaller than 0.25 (assuming an average inclination of $60^{\circ}$ ), and are likely to form AM CVn systems. These six systems also have the highest total mass $\left(M_{1}+M_{2}\right)$ in our sample. $\mathrm{J} 1233+1602$ is the most extreme case with $M_{1}+M_{2}=1.37 M_{\odot}$ for $i=60^{\circ}$. It is striking that the number of extreme mass ratio systems in our sample (six) is the same as the number of AM CVn systems found in the SDSS Data Relase 5 area (Roelofs et al. 2007). These systems are potential SNe .Ia progenitors (Bildsten et al. 2007).

Based on a magnitude-limited sample of six ELM WD mergers, Brown et al. (2010b) find that half of their sample has mass ratios of $\approx 0.15$ and they estimate a contribution factor of $\gtrsim 3 \%$ to the $\mathrm{AM} \mathrm{CVn}$ formation rate from the observed population of ELM WDs. The fraction of extreme mass ratio systems in our survey is also $50 \%$ (six out of 12 ELM WDs), supporting the conclusions reached by Brown et al. (2010b).

The other six ELM WD systems in our sample have mass ratios larger than 0.4. Kilic et al. (2010a) discuss various evolutionary scenarios for these objects. These systems will merge within a Hubble time and form a variety of exotic objects including underluminous $\mathrm{SNe}$, extreme helium stars ( $\mathrm{RCrB}$ ), or single helium-enriched subdwarf $\mathrm{O}$ stars. The merger rate

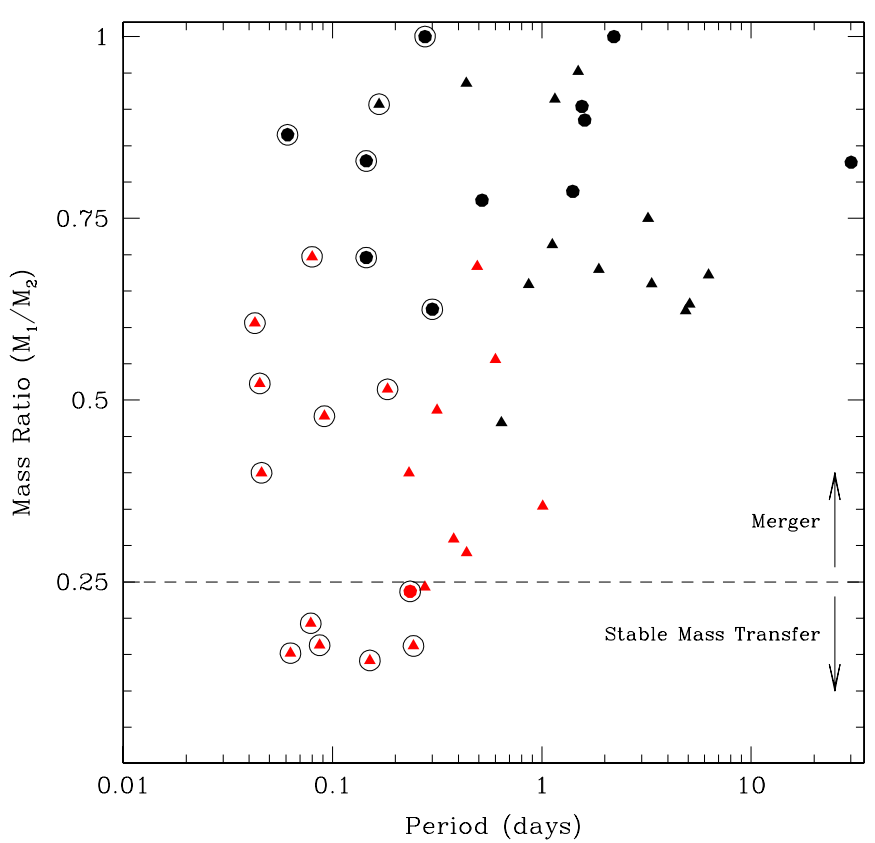

Figure 13. Mass ratios and periods for binary WD systems found in our ELM survey and the literature. The mass ratio limit for stable mass transfer is also indicated.

(A color version of this figure is available in the online journal.)

of ELM WDs is roughly the same as the observed rate of underluminous SNe (Brown et al. 2010b).

SDSS J1234-0228 is a unique ELM WD merger system. There is a $85 \%$ chance that the combined mass of the two stars in this system is lower than $0.45 M_{\odot}$. Therefore, within the next few billion years, this system will merge and likely form a a single low-mass WD. There are several scenarios to explain the presence of single low-mass $\left(\sim 0.4 M_{\odot}\right)$ WDs, including enhanced mass loss from metal-rich progenitors (Kilic et al. 2007c) and mass loss through common envelope evolution with a low-mass brown dwarf or planetary mass companion (Nelemans \& Tauris 1998). Single low-mass WDs can also form through mergers of two lower-mass WDs (Iben et al. 1997; Saio \& Jeffery 2000), though no such progenitors have been found until now. Here we have uncovered a potential progenitor system for a future single low-mass WD, J1234-0228.

About $10 \%$ of DA WDs are low mass (Liebert et al. 2005) and half of these low-mass WDs are single (Napiwotzki et al. 2004), corresponding to a space density of $2.5 \times 10^{5} \mathrm{kpc}^{-3}$ (Sion et al. 2009). The SDSS DR4 WD catalog covers 4783 square degrees with $g=15-20 \mathrm{mag}$ (Eisenstein et al. 2006). We estimate the local space density using the modified $1 / V_{\max }$ method and the disk model of Roelofs et al. (2007). Based on one system, J1234-0228, the space density of merging WD systems that can form single low-mass WDs is $1.1 \mathrm{kpc}^{-3}$. This number is likely uncertain by a factor of 10 due to incompleteness of the SDSS spectroscopic target selection. Nevertheless, it is several orders of magnitude smaller than the estimated space density of single low-mass WDs. Hence, this formation channel does not significantly contribute to the single low-mass WD population in the solar neighborhood.

\section{CONCLUSIONS}

Our MMT ELM survey has now discovered 20 short period binary WD systems, including 12 merger systems. Only six binary WD merger systems were known prior to our work. 
The ELM survey has now tripled the number of such systems. These merger systems provide potential formation channels for a variety of interesting objects including underluminous $\mathrm{SNe}$, AM CVn, extreme helium stars, single subdwarf stars, and now single low-mass WDs.

All but one of the 19 stars with $M \leqslant 0.25 M_{\odot}$ are in binaries, strongly arguing for a binary formation channel for ELM WDs. The combined sample of more than 40 short period WD systems found in our ELM survey and the literature demonstrate that the shortest period detached WD systems have $\approx 1 \mathrm{hr}$ orbital periods and they also have the lowest mass primaries. In addition, the lowest mass WDs in double WD systems have $M=0.17 M_{\odot}$. Lower-mass objects may be produced in such systems but are currently undetected in the SDSS and other spectroscopic surveys.

The sample of ELM WD merger systems will be significantly enlarged by our continuing ELM survey based on SDSS photometry. Such a magnitude-limited survey will not suffer from the biases present in our current sample, and will provide invaluable information on the merger rates of ELM WD systems and their contribution to underluminous SNe and AM CVn systems. Furthermore, a large sample of ELM WDs and population synthesis models will improve our understanding of the cooling models for ELM WDs.

We thank G. Nelemans for providing his binary WD population synthesis model results, S. Geier for the latest results from the SPY survey, and D. Koester for WD model spectra. Support for this work was provided by NASA through the Spitzer Space Telescope Fellowship Program, under an award from Caltech.

Facilities: MMT (Blue Channel Spectrograph), CXO (ACIS-S)

\section{APPENDIX}

\section{RADIAL VELOCITY DATA}

Table 5 presents the radial velocity measurements.

Table 5

Radial Velocity Measurements

\begin{tabular}{lcr}
\hline \hline \multicolumn{1}{c}{ Object } & \multicolumn{1}{c}{ HJD } & \multicolumn{1}{c}{$\begin{array}{c}v_{\text {helio }} \\
\left(\mathrm{km} \mathrm{s}^{-1}\right)\end{array}$} \\
\hline J0022+0031 & 732.722684 & $-72.2 \pm 30.3$ \\
$\ldots$ & 732.858519 & $22.4 \pm 18.8$ \\
$\ldots$ & 732.939955 & $21.2 \pm 36.0$ \\
$\ldots$ & 732.957466 & $-19.3 \pm 50.4$ \\
$\ldots$ & 733.728259 & $-45.4 \pm 19.5$ \\
$\ldots$ & 733.850239 & $15.3 \pm 11.6$ \\
$\ldots$ & 733.951397 & $-102.0 \pm 47.9$ \\
$\ldots$ & 1094.846527 & $-12.4 \pm 12.4$ \\
$\ldots$ & 1100.810977 & $59.7 \pm 15.6$ \\
$\ldots$ & 1100.827123 & $39.7 \pm 10.6$ \\
$\ldots$ & 1100.841278 & $45.5 \pm 26.9$ \\
$\ldots$ & 1100.858385 & $80.6 \pm 14.8$ \\
$\ldots$ & 1100.872447 & $74.6 \pm 19.8$ \\
$\ldots$ & 1100.890352 & $93.5 \pm 15.6$ \\
$\ldots$ & 1100.904427 & $31.7 \pm 16.6$ \\
$\ldots$ & 1100.919566 & $51.9 \pm 23.7$ \\
$\ldots$ & 1101.895459 & $19.9 \pm 22.9$ \\
$\ldots$ & 1101.906050 & $36.9 \pm 21.6$ \\
$\ldots$ & 1101.917786 & $17.5 \pm 21.0$ \\
$\ldots$ & 1102.904928 & $38.2 \pm 13.9$ \\
$\ldots$ & 1102.915530 & $33.1 \pm 26.3$
\end{tabular}

Table 5

(Continued)

\begin{tabular}{|c|c|c|}
\hline Object & $\begin{array}{c}\text { HJD } \\
-2450000\end{array}$ & $\begin{array}{c}v_{\text {helio }} \\
\left(\mathrm{km} \mathrm{s}^{-1}\right)\end{array}$ \\
\hline$\ldots$ & 1384.944307 & $100.6 \pm 29.7$ \\
\hline$\ldots$ & 1385.929157 & $-75.1 \pm 40.6$ \\
\hline$\ldots$ & 1387.925074 & $-14.8 \pm 72.2$ \\
\hline$\ldots$ & 1395.943892 & $-96.8 \pm 19.8$ \\
\hline$\ldots$ & 1396.936261 & $-87.0 \pm 17.1$ \\
\hline$\ldots$ & 1396.976913 & $-11.9 \pm 49.8$ \\
\hline J0022-1014 & 732.748459 & $-156.9 \pm 27.6$ \\
\hline$\ldots$ & 732.759199 & $-59.1 \pm 41.4$ \\
\hline$\ldots$ & 732.832753 & $-232.5 \pm 32.4$ \\
\hline$\ldots$ & 732.843424 & $-77.3 \pm 32.2$ \\
\hline$\ldots$ & 732.913470 & $-199.3 \pm 24.1$ \\
\hline$\ldots$ & 732.924142 & $13.5 \pm 20.7$ \\
\hline$\ldots$ & 733.781989 & $-198.3 \pm 44.7$ \\
\hline$\ldots$ & 733.872718 & $-125.5 \pm 47.9$ \\
\hline$\ldots$ & 1094.862244 & $25.5 \pm 14.1$ \\
\hline$\ldots$ & 1102.756314 & $89.0 \pm 31.1$ \\
\hline$\ldots$ & 1102.770423 & $67.9 \pm 31.6$ \\
\hline$\ldots$ & 1102.787923 & $-148.8 \pm 20.6$ \\
\hline$\ldots$ & 1102.801997 & $-214.7 \pm 22.9$ \\
\hline$\ldots$ & 1102.818895 & $33.5 \pm 18.9$ \\
\hline$\ldots$ & 1102.832969 & $59.5 \pm 15.6$ \\
\hline$\ldots$ & 1102.848212 & $46.9 \pm 18.2$ \\
\hline$\ldots$ & 1102.862274 & $-121.9 \pm 19.0$ \\
\hline$\ldots$ & 1102.876371 & $-148.9 \pm 33.8$ \\
\hline $\mathrm{J} 1224-0228$ & 917.805724 & $-1.6 \pm 6.2$ \\
\hline$\ldots$ & 922.728604 & $98.1 \pm 6.4$ \\
\hline$\ldots$ & 922.815478 & $111.6 \pm 5.8$ \\
\hline$\ldots$ & 922.904031 & $132.2 \pm 14.9$ \\
\hline$\ldots$ & 923.754131 & $-16.3 \pm 5.2$ \\
\hline$\ldots$ & 923.867625 & $-22.5 \pm 4.6$ \\
\hline$\ldots$ & 923.931236 & $6.6 \pm 7.9$ \\
\hline$\ldots$ & 924.850731 & $52.6 \pm 13.4$ \\
\hline$\ldots$ & 924.857965 & $-61.9 \pm 17.8$ \\
\hline$\ldots$ & 1275.829077 & $136.3 \pm 10.1$ \\
\hline$\ldots$ & 1275.834841 & $73.9 \pm 11.9$ \\
\hline$\ldots$ & 1275.841936 & $17.8 \pm 10.5$ \\
\hline$\ldots$ & 1275.847700 & $-20.6 \pm 7.8$ \\
\hline$\ldots$ & 1275.853441 & $-15.4 \pm 9.3$ \\
\hline$\ldots$ & 1275.860270 & $-13.8 \pm 11.7$ \\
\hline$\ldots$ & 1275.866022 & $-21.6 \pm 11.9$ \\
\hline$\ldots$ & 1275.871763 & $-27.3 \pm 8.4$ \\
\hline$\ldots$ & 1275.919252 & $146.8 \pm 11.0$ \\
\hline$\ldots$ & 1275.925560 & $103.6 \pm 12.5$ \\
\hline $\mathrm{J} 1625+3632$ & 922.872132 & $-90.0 \pm 12.8$ \\
\hline$\ldots$ & 922.957182 & $-146.1 \pm 9.7$ \\
\hline$\ldots$ & 923.002704 & $-39.0 \pm 9.6$ \\
\hline$\ldots$ & 923.837051 & $-160.2 \pm 10.4$ \\
\hline$\ldots$ & 923.912980 & $-90.8 \pm 8.4$ \\
\hline$\ldots$ & 923.956569 & $-54.9 \pm 7.6$ \\
\hline$\ldots$ & 924.004812 & $-73.7 \pm 16.0$ \\
\hline$\ldots$ & 925.000912 & $-134.7 \pm 11.0$ \\
\hline$\ldots$ & 925.012835 & $-140.7 \pm 21.9$ \\
\hline$\ldots$ & 1276.936718 & $-12.2 \pm 14.2$ \\
\hline$\ldots$ & 1276.943038 & $-25.0 \pm 17.5$ \\
\hline$\ldots$ & 1276.951372 & $-11.3 \pm 17.6$ \\
\hline$\ldots$ & 1276.957668 & $-57.6 \pm 17.7$ \\
\hline$\ldots$ & 1276.964011 & $-60.2 \pm 13.3$ \\
\hline$\ldots$ & 1276.972808 & $-7.3 \pm 24.7$ \\
\hline$\ldots$ & 1276.979128 & $-34.2 \pm 27.0$ \\
\hline$\ldots$ & 1276.985447 & $-50.1 \pm 26.9$ \\
\hline$\ldots$ & 1384.783927 & $-51.8 \pm 20.6$ \\
\hline$\ldots$ & 1385.738969 & $-59.6 \pm 11.9$ \\
\hline$\ldots$ & 1396.823036 & $-23.7 \pm 42.7$ \\
\hline
\end{tabular}


Table 5

(Continued)

\begin{tabular}{|c|c|c|}
\hline Object & $\begin{array}{c}\text { HJD } \\
-2450000\end{array}$ & $\begin{array}{c}v_{\text {helio }} \\
\left(\mathrm{km} \mathrm{s}^{-1}\right)\end{array}$ \\
\hline $\mathrm{J} 2252-0056$ & 732.661721 & $-33.0 \pm 8.4$ \\
\hline$\ldots$ & 732.706500 & $-52.3 \pm 13.5$ \\
\hline$\ldots$ & 732.786660 & $-40.0 \pm 11.8$ \\
\hline$\ldots$ & 732.872943 & $-17.0 \pm 16.2$ \\
\hline$\ldots$ & 733.653315 & $-24.9 \pm 16.9$ \\
\hline$\ldots$ & 733.752698 & $4.8 \pm 13.1$ \\
\hline$\ldots$ & 733.761692 & $-39.8 \pm 13.9$ \\
\hline$\ldots$ & 733.829687 & $-18.2 \pm 18.7$ \\
\hline$\ldots$ & 733.892232 & $-11.5 \pm 11.7$ \\
\hline$\ldots$ & 1094.801506 & $-22.9 \pm 7.3$ \\
\hline$\ldots$ & 1100.666435 & $-18.0 \pm 16.4$ \\
\hline$\ldots$ & 1100.681967 & $4.7 \pm 9.2$ \\
\hline$\ldots$ & 1100.696261 & $-14.5 \pm 8.9$ \\
\hline$\ldots$ & 1100.712232 & $34.4 \pm 8.3$ \\
\hline$\ldots$ & 1100.726399 & $-12.9 \pm 9.5$ \\
\hline$\ldots$ & 1100.742289 & $1.4 \pm 8.7$ \\
\hline$\ldots$ & 1100.756386 & $26.4 \pm 10.8$ \\
\hline$\ldots$ & 1100.770645 & $-29.4 \pm 15.4$ \\
\hline$\ldots$ & 1100.781270 & $-29.9 \pm 11.5$ \\
\hline$\ldots$ & 1101.650617 & $-35.3 \pm 15.1$ \\
\hline$\ldots$ & 1101.659471 & $20.6 \pm 13.9$ \\
\hline$\ldots$ & 1101.672399 & $-118.1 \pm 16.3$ \\
\hline$\ldots$ & 1101.682989 & $27.1 \pm 16.7$ \\
\hline$\ldots$ & 1101.698706 & $-35.8 \pm 13.6$ \\
\hline$\ldots$ & 1101.709307 & $-2.8 \pm 16.8$ \\
\hline$\ldots$ & 1101.719897 & $-33.6 \pm 17.3$ \\
\hline$\ldots$ & 1102.645214 & $-86.1 \pm 19.9$ \\
\hline$\ldots$ & 1102.655804 & $28.9 \pm 20.7$ \\
\hline$\ldots$ & 1102.667655 & $-65.7 \pm 23.2$ \\
\hline$\ldots$ & 1102.678245 & $-63.8 \pm 15.3$ \\
\hline $\mathrm{J} 2345-0102$ & 732.689649 & $-219.3 \pm 27.1$ \\
\hline$\ldots$ & 732.799579 & $-130.2 \pm 38.3$ \\
\hline$\ldots$ & 732.810239 & $-94.4 \pm 35.0$ \\
\hline$\ldots$ & 732.889312 & $-114.6 \pm 30.3$ \\
\hline$\ldots$ & 733.697952 & $-129.3 \pm 31.9$ \\
\hline$\cdots$ & 733.711030 & $-159.5 \pm 53.8$ \\
\hline$\ldots$ & 733.809108 & $-142.7 \pm 24.4$ \\
\hline$\ldots$ & 733.928533 & $-153.0 \pm 47.3$ \\
\hline$\ldots$ & 1094.830335 & $-175.5 \pm 28.8$ \\
\hline$\ldots$ & 1101.735791 & $-139.1 \pm 59.8$ \\
\hline$\ldots$ & 1101.751161 & $-242.8 \pm 64.4$ \\
\hline$\ldots$ & 1101.765224 & $-243.8 \pm 41.6$ \\
\hline$\ldots$ & 1101.786080 & $-109.0 \pm 31.3$ \\
\hline$\ldots$ & 1101.800154 & $-97.8 \pm 23.5$ \\
\hline$\ldots$ & 1101.816149 & $-199.4 \pm 40.1$ \\
\hline$\ldots$ & 1101.830211 & $-138.7 \pm 25.3$ \\
\hline$\cdots$ & 1101.846137 & $-109.9 \pm 26.4$ \\
\hline$\ldots$ & 1101.860199 & $-138.8 \pm 22.3$ \\
\hline$\ldots$ & 1102.701680 & $-136.8 \pm 29.2$ \\
\hline$\ldots$ & 1102.719307 & $-95.9 \pm 65.7$ \\
\hline$\ldots$ & 1102.736402 & $-135.1 \pm 46.7$ \\
\hline
\end{tabular}

\section{REFERENCES}

Augusteijn, T., van der Hooft, F., de Jong, J. A., \& van Paradijs, J. 1996, A\&A 311,889

Agüeros, M. A., Camilo, F., Silvestri, N. M., Kleinman, S. J., Anderson, S. F., \& Liebert, J. W. 2009a, ApJ, 697, 283

Agüeros, M. A., et al. 2009, ApJ, 700, L123

Allende Prieto, C., Hubeny, I., \& Smith, J. A. 2009, MNRAS, 396, 759

Badenes, C., Mullally, F., Thompson, S. E., \& Lupton, R. H. 2009, ApJ, 707, 971

Bassa, C. G., van Kerkwijk, M. H., Koester, D., \& Verbunt, F. 2006, A\&A, 456, 295

Beloborodov, A. M. 2002, ApJ, 566, L85
Bildsten, L., Shen, K. J., Weinberg, N. N., \& Nelemans, G. 2007, ApJ, 662, L95 Bogdanov, S., Grindlay, J. E., \& Rybicki, G. B. 2006, ApJ, 648, L55

Branch, D., Livio, M., Yungelson, L. R., Boffi, F. R., \& Baron, E. 1995, PASP, 107, 1019

Brown, J. M., Kilic, M., Brown, W. R., \& Kenyon, S. J. 2010, ApJ, submitted

Brown, W. R., Geller, M. J., Kenyon, S. J., \& Kurtz, M. J. 2006, ApJ, 647, 303

Brown, W. R., Kilic, M., Allende Prieto, C., \& Kenyon, S. J. 2010a, ApJ, 723, 1072

Brown, W. R., Kilic, M., Allende Prieto, C., \& Kenyon, S. J. 2010b, MNRAS, in press

Dickey, J. M., \& Lockman, F. J. 1990, ARA\&A, 28, 215

Di Stefano, R. 2010, ApJ, 719, 474

Driebe, T., Schoenberner, D., Bloecker, T., \& Herwig, F. 1998, A\&A, 339, 123

Eisenstein, D. J., et al. 2006, ApJS, 167, 40

Gehrels, N. 1986, ApJ, 303, 336

Geier, S., Heber, U., Kupfer, T., \& Napiwotzki, R. 2010, A\&A, 515, A37

Hansen, B. M. S. 1999, ApJ, 520, 680

Heber, U., Edelmann, H., Lisker, T., \& Napiwotzki, R. 2003, A\&A, 411, L477

Heinke, C. O., Grindlay, J. E., Edmonds, P. D., Cohn, H. N., Lugger, P. M. Camilo, F., Bogdanov, S., \& Freire, P. C. 2005, ApJ, 625, 796

Hogg, D. W., Blanton, M. R., Roweis, S. T., \& Johnston, K. V. 2005, ApJ, 629, 268

Iben, I., Jr., \& Tutukov, A. V. 1984, ApJS, 54, 335

Iben, I., Jr., Tutukov, A. V., \& Yungelson, L. R. 1997, ApJ, 475, 291

Kawka, A., Vennes, S., \& Vaccaro, T. R. 2010, A\&A, 516, L7

Kenyon, S. J., \& Garcia, M. R. 1986, AJ, 91, 125

Kilic, M., Allende Prieto, C., Brown, W. R., \& Koester, D. 2007, ApJ, 660, 1451

Kilic, M., Brown, W. R., Allende Prieto, C., Pinsonneault, M. H., \& Kenyon, S. J. 2007b, ApJ, 664, 1088

Kilic, M., Stanek, K. Z., \& Pinsonneault, M. H. 2007, ApJ, 671, 761

Kilic, M., Brown, W. R., Allende Prieto, C., Swift, B., Kenyon, S. J., Liebert, J., \& Agüeros, M. A. 2009, ApJ, 695, L92

Kilic, M., Brown, W. R., \& McLeod, B. 2010, ApJ, 708, 411

Kilic, M., Brown, W. R., Allende Prieto, C., \& Kenyon, S. J. 2010, ApJ, 716, 122

Kilic, M., Allende Prieto, C., Brown, W. R., Agüeros, M. A., Kenyon, S. J., \& Camilo, F. 2010, ApJ, 721, L158

Kotak, R. 2008, in ASP Conf. Ser. 401, RS Ophiuchi (2006) and the Recurrent Nova Phenomenon, ed. A. Evans et al. (San Francisco, CA: ASP), 150

Kromer, M., Sim, S. A., Fink, M., Röpke, F. K., Seitenzahl, I. R., \& Hillebrandt, W. 2010, ApJ, 719, 1067

Kroupa, P., \& Tout, C. A. 1997, MNRAS, 287, 402

Kulkarni, S. R., \& van Kerkwijk, M. H. 2010, ApJ, 719, 1123

Kurtz, M. J., \& Mink, D. J. 1998, PASP, 110, 934

Liebert, J., Bergeron, P., Eisenstein, D., Harris, H. C., Kleinman, S. J., Nitta, A. \& Krzesinski, J. 2004, ApJ, 606, L147

Liebert, J., Bergeron, P., \& Holberg, J. B. 2005, ApJS, 156, 47

Marsh, T. R. 1995, MNRAS, 275, L1

Marsh, T. R., Gaensicke, B. T., Steeghs, D., Southworth, J., Koester, D., Harris, V., \& Merry, L. 2010, ApJ, submitted (arXiv:1002.4677)

Massey, P., Strobel, K., Barnes, J. V., \& Anderson, E. 1988, ApJ, 328, 315

Maxted, P. F. L., Marsh, T. R., \& Moran, C. K. J. 2000, MNRAS, 319, 305

Mullally, F., Badenes, C., Thompson, S. E., \& Lupton, R. 2009, ApJ, 707, L51

Munn, J. A., et al. 2004, AJ, 127, 3034

Napiwotzki, R., Edelmann, H., Heber, U., Karl, C., Drechsel, H., Pauli, E.-M., \& Christlieb, N. 2001, A\&A, 378, L17

Napiwotzki, R., et al. 2004a, in ASP Conf. Ser. 318, Spectroscopically and Spatially Resolving the Components of the Close Binary Stars, ed. R. W. Hilditch, H. Hensberge, \& K. Pavlovski (San Francisco, CA: ASP), 402

Napiwotzki, R., et al. 2007, in ASP Conf. Ser. 372, 15th European Workshop on White Dwarfs, ed. R. Napiwotzki \& M. R. Burleigh (San Francisco, CA: ASP), 387

Nelemans, G., \& Tauris, T. M. 1998, A\&A, 335, L85

Nelemans, G., Yungelson, L. R., Portegies Zwart, S. F., \& Verbunt, F. 2001, A\&A, 365, 491

Nelemans, G., Yungelson, L. R., \& Portegies Zwart, S. F. 2004, MNRAS, 349, 181

Nelemans, G., et al. 2005, A\&A, 440, 1087

Panei, J. A., Althaus, L. G., Chen, X., \& Han, Z. 2007, MNRAS, 382, 779

Roelofs, G. H. A., Groot, P. J., Steeghs, D., Marsh, T. R., \& Nelemans, G. 2007, MNRAS, 382, 1643

Saio, H., \& Jeffery, C. S. 2000, MNRAS, 313, 671

Serenelli, A. M., Althaus, L. G., Rohrmann, R. D., \& Benvenuto, O. G. 2001, MNRAS, 325, 607

Serenelli, A. M., Althaus, L. G., Rohrmann, R. D., \& Benvenuto, O. G. 2002, MNRAS, 337, 1091 
Sim, S. A., Röpke, F. K., Hillebrandt, W., Kromer, M., Pakmor, R., Fink, M., Ruiter, A. J., \& Seitenzahl, I. R. 2010, ApJ, 714, L52

Sion, E. M., Holberg, J. B., Oswalt, T. D., McCook, G. P., \& Wasatonic, R. 2009, AJ, 138, 1681

Steinfadt, J. D. R., Kaplan, D. L., Shporer, A., Bildsten, L., \& Howell, S. B. 2010, ApJ, 716, L146 van Kerkwijk, M. H., Bergeron, P., \& Kulkarni, S. R. 1996, ApJ, 467, L89 Vennes, S., Kawka, A., Vaccaro, T. R., \& Silvestri, N. M. 2009, A\&A, 507, 1613

Voges, W., et al. 1999, A\&A, 349, 389

Zavlin, V. E., Pavlov, G. G., Sanwal, D., Manchester, R. N., Trümper, J., Halpern, J. P., \& Becker, W. 2002, ApJ, 569, 894

Zavlin, V. E. 2006, ApJ, 638, 951 